\title{
$\mathrm{KJCCM}$
}

Korean J Crit Care Med

\section{Spontaneous Lumbar Artery Bleeding and Retroperitoneal Hematoma in a Patient Treated with Continuous Renal Replacement Therapy}

Jin Kyung Park, M.D. ${ }^{1}$, Sung Ho Kim, M.D. ${ }^{1}$, Hee Jin Kim, M.D. ${ }^{2}$, and Duk Hyun Lee, M.D. ${ }^{1}$

${ }^{1}$ Department of Internal Medicine, ${ }^{2}$ Department of Radiology, Daegu Fatima Hospital, Daegu, Korea

2015 November 30(4):318-322 / http://dx.doi.org/10.4266/kjccm.2015.30.4.318

We found an error in this article. The Fig. 2B should be corrected as following:

In this article, on page 319, Fig. 2B has an error. The position of its arrow was wrong.

We present the correct figure as below:

Before correction

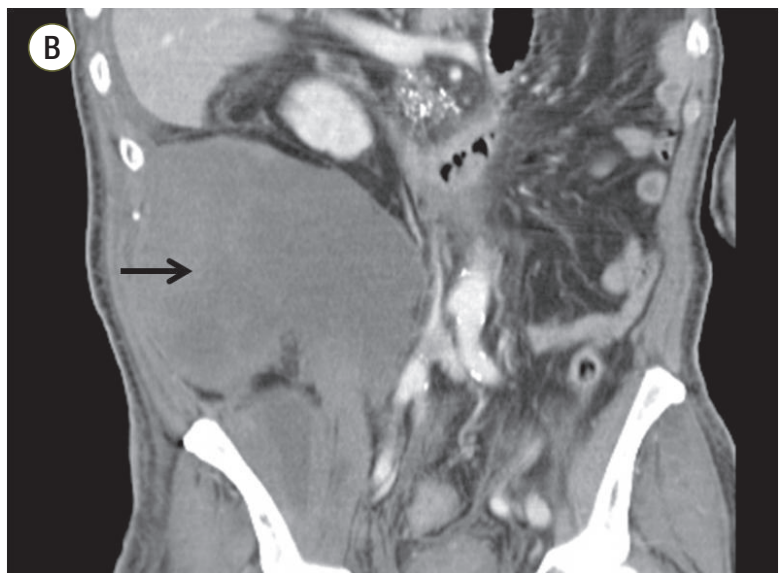

After correction

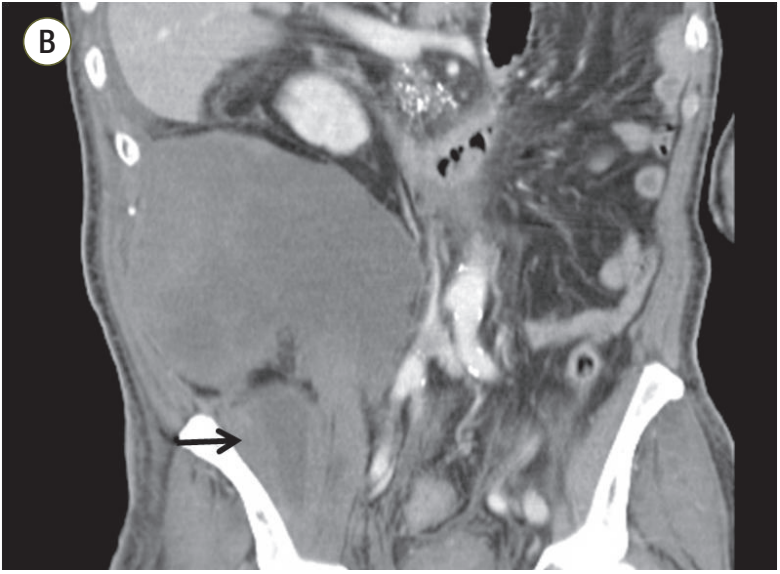

We apologize for any inconvenience that may have caused. 